\title{
DE LA COGNICIÓN AL DISCURSO: EL EFECTO DE LA PROMINENCIA COGNITIVA Y LA INFORMATIVIDAD TEXTUAL EN EL ESTUDIO DE LA VARIACIÓN DE LOS SUJETOS PRONOMINALES
}

\author{
MARÍA JOSÉ SERRANO \\ Universidad de la Laguna \\ mjserran@ull.edu.es
}

\begin{abstract}
Resumen
La prominencia cognitiva se basa en el proceso de atención, modelado en términos de activación mental de estructuras conceptuales. Por su parte, el concepto de informatividad se relaciona con el nivel de procesamiento mental que requiere un texto en función del grado de novedad o de imprevisibilidad que tiene para sus receptores. De acuerdo con ello, en español, la variación entre la expresión (preverbal y posverbal) y la omisión del sujeto ( $Y o$ vengo/ $\varnothing$ vengo) puede ser una manifestación formal de las variaciones en la prominencia cognitiva de los referentes. La idea de que la informatividad textual guarda cierta relación con la colocación de las unidades en la cláusula puede aplicarse al estudio de la posición preverbal o posverbal del sujeto pronominal (Yo vengo/Vengo yo). Bajo una metodología cualitativa, se considera que estas posibilidades variables, fundamentadas cognitivamente y que ofrecen posibilidades para la construcción de un significado socioestilístico, constituyen uno de los objetivos del enfoque que aplicamos para el estudio de la variación en los sujetos pronominales en español.
\end{abstract}

PALABRAS CLAVE: prominencia cognitiva, informatividad textual, sujetos pronominales, variación, sintaxis.

\begin{abstract}
Linguistic units suggest semantic frames that conceptualize meanings and target concrete interpretations. Cognitive salience is one of these conceptualizations based on the attention process shaped by mental accesibility of structures. By its part, informativeness is a textual discursive device related to the level of mental effort needed by speakers/hearers in processing texts by means of its new or unexpected nature. In Spanish language, variation between expression and omission of pronominal subjects (Yo vengo/ Ø vengo; I come) can be explained as formal signs of cognitive salience of its referents. Informativeness is also related to the position of elements in the sentence, so preverbal or posverbal position of these pronouns (Yo vengo/Vengo yo) may be analyzed with such textual procedure. By a cualitative methodology, the fact that these variants contribute to create sociostylistic meanings in discourse and communicative interaction is the main goal of our ongoing research of variation of pronominal subjects in Spanish.
\end{abstract}

KEY WORDS: Salience, textual informativeness, pronominal subjects, variation, syntax.

\section{Introducción}

En este trabajo se propone la descripción de dos herramientas analíticas cognitivas y textuales utilizadas para explicar la variación de los sujetos pronominales en español, esto es, la prominencia cognitiva y la informatividad textual. El propósito de esta inves- 
tigación no es solamente señalar la naturaleza teórica de dichas nociones, sino la utilidad que ofrecen para el estudio de este fenómeno variable. Para ello, en primer lugar se realizará un resumen crítico de la prominencia cognitiva y de la informatividad textual y, en segundo, se presentarán varios ejemplos de cómo se aplican en la definición, análisis y determinación de las variantes del sujeto pronominal en español a partir de los resultados obtenidos en algunas investigaciones anteriores. La metodología empleada es cualitativa, ya que ofrece una aplicación hermenéutica de las mencionadas nociones al estudio de la variación como paso previo para emprender el correspondiente análisis estadístico.

1. La prominencia como operación de conceptualización cognitiva. Algunas precisiones teóricas y su aplicación a la expresión (preverbal o posverbal) y omisión del sujeto en español

La variación morfosintáctica se produce en virtud de la naturaleza icónica, simbólica y gradual de la gramática (cf. Langacker, 1991, 2009; Croft y Cruse, 2008); las construcciones y formas gramaticales conducen a la creación de cierto significado que puede ser utilizado con fines e intenciones concretos por distintos hablantes y en distintas situaciones comunicativas.

Para perfilar las características cognitivas de la variación gramatical es útil centrarse en una de las principales hipótesis de la lingüística cognitiva, consistente en que el uso del lenguaje está marcado por operaciones de conceptualización: todas las unidades lingüísticas evocan un marco semántico que conceptualiza su significado y lo orienta hacia una interpretación determinada. Con ello se cuestiona la identidad de significado o la sinonimia en el lenguaje y, paralelamente, se invalida la semántica de carácter veritativo-condicional o la remisión al referente para su descripción (Croft y Cruse, 2008: 65; Langacker, 1999). Para ser realmente cognitivas, las operaciones de conceptualización han de tener un correlato en algún proceso psicológico general. Además, son también conceptualizaciones de la experiencia que se quiere comunicar, de modo que se corresponden con uno de los postulados básicos de la lingüística cognitiva: el experiencialismo o la corporeización de los significados lingüísticos (Vega, 2005; Lakoff, 1987; Talmy, 1988). Ello se aparta también de una concepción de la lengua como independiente de la realidad circundante y afirma su condición inherentemente social, lo cual determina que el lenguaje es parte de una capacidad cognitiva más general, de base fundamentalmente cerebral (López García, 2010).

Estos principios resultan de gran utilidad para el estudio de la variación morfosintáctica, toda vez que se ha comprobado que para su estudio ya no resulta eficiente basar 
la investigación en la búsqueda de la identidad significativa de las formas (Serrano, 2011a). Antes bien, es precisamente la diferencia de significado lo que puede ayudar a comprender el funcionamiento de las variantes formales y a relacionarlo con distintos aspectos sociales y comunicativos (Aijón Oliva y Serrano, 2010a; Serrano y Aijón Oliva, 2011) $)^{1}$.

Una de las operaciones de conceptualización que resulta útil para estudiar la variación como coexistencia de opciones significativas que pueden crear estilos comunicativos es la prominencia cognitiva. Su base reside en el proceso de atención, concepto procedente de la psicología cognitiva y modelado en términos de activación mental de estructuras conceptuales. En la lengua existen unidades que pueden estar más fijadas en la atención del hablante/oyente, y por ello resultar más prominentes, lo cual conduce a que su significado y su función se conceptualicen de un modo u otro y a que, al mismo tiempo, puedan ser comparables o no a otras estructuras.

La prominencia se puede analizar teniendo en cuenta dos aspectos: el foco y el ámbito de atención ${ }^{2}$. El primero es el punto sobre el que se centra la atención, mientras que el segundo consiste en aquello que rodea al foco, a partir del cual las unidades pueden ser más o menos accesibles. De hecho, la noción de prominencia está estrechamente relacionada con la accesibilidad de los referentes (Ariel, 1990; Chafe, 1976; Gundel y Fretheim, 2009); así, aquel referente que está activado en el foco de la atención del hablante resultará más accesible, es decir, estará activado en la mente del hablante. Por lo tanto, un elemento lingüístico será más prominente si obtiene la mayor accesibilidad en un punto concreto del discurso, aunque se considera que las unidades prominentes (pronombres, determinantes, deícticos, etc.) suelen estar activadas en la mente del hablante, independientemente del contexto. Por ejemplo, en la frase Comemos a las dos, el sujeto (pronominal o léxico) correlacionado con la desinencia verbal nosotros es prominente o accesible independientemente del contexto. En otro enunciado como María fue a la farmacia, espero que no esté enferma, el sujeto de esté resulta prominente (ella) y además su referente léxico, que ya ha sido mencionado previamente (María), es accesible por medio de la desinencia verbal. En suma, esta noción viene impuesta de

\footnotetext{
${ }^{1}$ Es cierto que la función de la conceptualización en el lenguaje se hace más que evidente cuando en un mismo idioma existen expresiones alternativas para lo que parecen ser situaciones equivalentes desde el punto de vista veritativo-funcional (Croft y Cruse, 2008: 65); es decir, las variantes gramaticales en sentido tradicional.

2 Estos términos son asimilables a los que maneja Langacker (2009: 48) de punto de referencia (que sería análogo al foco de atención) y dominio (que constituiría el ámbito de atención).
} 
alguna forma por las estructuras lingüísticas usadas para representar la escena comunicativa (Langacker, 2009: 112).

La prominencia cognitiva se corresponde, pues, con un determinado nivel de activación o accesibilidad del referente. En una línea teórica similar, aunque sin efectuar remisión expresa al plano cognitivo ${ }^{4}$, las escuelas funcionalistas utilizan los términos topicalización y tematicidad (Halliday, 1967; Givón, 2001).

Desde perspectivas pragmáticas, el estatus informativo de los elementos textuales también puede relacionarse con la prominencia. Así, los elementos ya conocidos a través del discurso previo serán, por lo general, más prominentes y tenderán a situarse al inicio de la cláusula ${ }^{6}$ (Prince, 1981: 230; Levinson, 1983: 88; Chafe, 1976). Sin embargo, la prominencia cognitiva no se relaciona exclusivamente con el estatus nuevo/conocido del contenido proposicional, ni debería definirse a partir de él.

De hecho, una de las soluciones más comunes para caracterizar la prominencia o los conceptos funcionales análogos de topicalización o tematicidad es aludir a la transmisión de contenidos ya conocidos y a la posición sintáctica inicial. Aunque estos aspectos sean recurrentes en la mayor parte de las descripciones, la prominencia cognitiva añade una noción basada en el foco de atención o accesibilidad de los referentes en cuanto a su grado de activación en la conciencia de los hablantes, algo que no parece ser tenido en cuenta (al menos de forma explícita) en los enfoques no cognitivos. Lambrecht (1998:

\footnotetext{
${ }^{3}$ La prominencia cognitiva no deja de ser una noción amplia, pero su utilidad dependerá de cómo se relacione con otros factores o se aplique a algunos de forma concreta (Geeraerts, Dirven y Taylor, 2006: 37-38). Una vertiente de su aplicación es la relacionada con aspectos de organización sintáctica de la información, como la que aquí se expone (Evans y Pourcel, 2009).

${ }^{4}$ El tratamiento de la cognición en las escuelas funcionalistas parece estar en un segundo plano, aunque a juzgar por los temas tratados por ellas (como la topicalización o tematización) su estudio debería figurar entre sus preocupaciones fundamentales. Así lo sostiene Langacker (1999: 22), aludiendo a la necesidad de una complementariedad entre los enfoques funcionales y los propiamente cognitivistas. ${ }^{5}$ Aun así, Langacker (1991: 306) reconoce que el término topicalidad en la gramática cognitiva puede recibir una interpretación simple, admitiendo la diferente secuenciación que pueden tener los elementos en una cláusula y su naturaleza semántica. De esta forma, un sujeto situado al inicio de la frase puede ser topical y al mismo tiempo exhibir un nivel muy elevado de prominencia. Tal sería el caso de la frase Le gusta el chocolate, donde le ocupa la posición habitual del sujeto (topical) y chocolate la de complemento directo, pese a que el verbo no es transitivo. Sin embargo, considera que es un término más encaminado a estudiar cuestiones de organización discursiva 'extrínseca' que estrictamente cognitivas. Véase Langacker (2004: 21-60) para una detallada comparación entre las nociones de tópico y punto de referencia.

${ }^{6}$ Numerosos estudios han apuntado a la relación entre la información conocida y la accesibilidad o prominencia; Giora (2003) realiza un análisis exhaustivo de cómo las palabras con significado conocido o accesible suelen ser relevantes para la comprensión y la producción del lenguaje.
} 
160) señala que el concepto de tópico viene definido por su relación con otros referentes de contenidos proposicionales próximos y de la información contextual compartida entre hablante y oyente (Prince, 1981), lo cual difiere esencialmente de las nociones de accesibilidad e identificabilidad.

De acuerdo con lo anterior, podría afirmarse que, en español, la variación entre la expresión y la omisión del sujeto puede ser una manifestación formal de las variaciones en la prominencia cognitiva de los referentes. Cuando el sujeto está activado en el foco de atención, es accesible o prominente y no es necesaria su formulación (o el hablante no considera que lo sea para sus propósitos comunicativos) ${ }^{7}$.

La omisión del sujeto, como variante de la expresión (y de la colocación del sujeto expreso en la cláusula), constituye una opción comunicativa para el hablante; de modo que, si bien en algunos casos la omisión parece obligatoria por la propia prominencia del referente (María se ha comprado una casa y tiene que pagar la hipoteca), en otros el hablante tiene la opción de expresarlo u omitirlo, consiguiendo así un significado concreto en el discurso (María se ha comprado una casa y ella tiene que pagar la hipoteca). Cuando el pronombre se expresa, se supone que su referente posee un nivel más bajo de prominencia y que existe alguna necesidad o intención discursivo-cognitiva que induce su expresión formal, que se pone de manifiesto en la interacción dando lugar a distintos significados que crean estilos comunicativos. Esta posibilidad variable, fundamentada cognitivamente y que ofrece recursos para la construcción de significados socioestilísticos, constituye el objetivo de este enfoque para el estudio de la variación sintáctica ${ }^{8}$.

Así pues, es posible proponer una matriz de prominencia con las variantes de expresión y omisión del sujeto, basada en ese nivel de atención o accesibilidad gradual que poseen en el discurso los referentes.

\begin{tabular}{lcc}
\hline & Sujeto omitido & Sujeto expreso \\
\hline Prominencia cognitiva & + prominente & - prominente \\
\hline Tabla 1. Gradualidad de la prominencia en el sujeto expreso y omitido
\end{tabular}

El sujeto omitido suele ser fácilmente identificable, bien a través de las desinencias verbales (lo que de entrada otorga mayor prominencia a los sujetos de primera o segunda persona) o bien a través de su previa mención en el discurso.

\footnotetext{
${ }^{7}$ De hecho, la variación lingüística ha de entenderse como la posibilidad de elegir una forma sobre otras para llevar a cabo determinados fines comunicativos, como ya hemos avanzado.

${ }^{8}$ Este trabajo forma parte del desarrollo del proyecto de I+D "Los estilos de comunicación y sus bases cognitivas en el estudio de la variación sintáctica en español” (FFI2009-07181/FILO).
} 
(1) Ø Quiero decirles que ya el TDT de San Roque en La Laguna está operativo len el área metropolitana yo les recomiendo\que se vayan $\emptyset$ olvidando de la televisión analógica $\backslash y$ para que nos $\varnothing$ puedan ver Igracias a Retevisión\vayan Ø sintonizándonos en TDT\en toda el área metropolitana\y en el sur nos pueden ver\pero en el 90\% de la isla $\varnothing$ no van a poder ver sin TDT $\varnothing$ tienen repetidores en Izaña \en Los Campitos $\backslash y$ en La Corona de San Roquely esto no tiene nada que ver con la Unelco\pásense $\varnothing$ a la TDT\Ø les recomiendo que cuanto más pronto mejor $($ CCEC $<$ Ma10Scan $>)$

Del mismo modo que el sujeto expreso es menos prominente que el omitido, es necesario determinar el nivel de prominencia relativa que adquieren las variantes preverbal y posverbal del primero.

En primer lugar, hay que tener en cuenta que en la lengua española el orden sintáctico prototípico es el que presenta al sujeto en posición preverbal SVO 9 . De acuerdo con estos esquemas, y aceptando que el sujeto es de entrada la perspectiva primaria desde la que se contempla una escena discursiva (Dik, 1981: 71; García, 2009: cap. 2), es fácil suponer que el grado de activación mental o prominencia que pueda tener el sujeto en un orden natural y prototípico (SV) será mayor que en una variación sobre dicho orden prototípico (VS). Que VS sea la forma marcada o no prototípica conduce a pensar que la posición posverbal no está tan accesible en el foco de atención del hablante, es decir, no posee naturalmente la capacidad de atraer la atención y potenciar la prominencia de los elementos (Croft y Cruse, 2008: 73) ${ }^{10}$. A ello se suma la infrecuencia, novedad o imprevisibilidad del citado esquema VS. Algunos autores han notado que la posición posverbal del sujeto pronominal es muy poco frecuente en la lengua española (Bentivoglio, 1988, 1989; Aijón Oliva y Serrano, 2010b; Serrano y Aijón Oliva, 2011; Serrano, 2012; Posio, 2012).

Otro aspecto que podría apoyar la menor prominencia del sujeto posverbal es su asimilación a las características formales y semánticas de los objetos sintácticos (Alonso Cortés, 2002:319). La relación entre la transitividad de la cláusula y la posición del suje-

\footnotetext{
9 Desde Nebrija, la tradición gramatical tiende a convenir que el orden SVO es el más característico de la lengua española. Gili Gaya es uno de los autores que más atención dedican a este aspecto, señalando que el sujeto suele ocupar la primera posición porque es el elemento que adquiere una mayor relevancia (1976: 85). Al respecto, véase también Fried (2009).

${ }^{10}$ Es posible que un sujeto posverbal, a través de la continuidad textual, adquiera prominencia cognitiva y se convierta en accesible, con lo cual pueda ser omitido en secuencias siguientes. No obstante, si sigue apareciendo expreso y en posición posverbal aunque el contexto señale que está activado o es accesible, habrá que suponer alguna motivación discursivo-pragmática o estilística por parte del hablante.
} 
to ha sido observada por algunos estudiosos. Delbecque $(2005: 4,9)$ concluye que en construcciones transitivas es más frecuente el sujeto antepuesto, mientras que en las intransitivas predomina la tendencia a la posposición ${ }^{11}$. Asimismo, el sujeto pospuesto tenderá a interpretarse menos como agente o iniciador del proceso o acción denotados por el verbo y más como afectado por estos.

En un estudio de los verbos que suelen coaparecer con una u otra posición del sujeto se ha podido concluir que, de forma general y siguiendo la clasificación de Hopper y Thompson (1980: 252), la posición preverbal asumiría algunos de los rasgos de la transitividad prototípica o alta (cf. Serrano, 2012). Por otra parte, dado que la colocación posverbal del sujeto supone una ruptura del esquema prototípico de la oración (SVO) y de la correspondiente transitividad alta, esto podría significar cognitivamente una objetualización del sujeto y una tendencia de la cláusula a asumir significados informativos. La posición posverbal se conceptualizaría en el ámbito, no en el foco de atención; por lo tanto, no sería prominente, debido a sus características de marcadez y objetualización sintáctica, puede adoptar rasgos textuales relacionados con la informatividad textual. Esta posición es susceptible de exigir un mayor esfuerzo y procesamiento interpretativos, al ser la menos probable o prototípica y presentar generalmente los contenidos como inesperados, imprevistos y, con ello, más realzados o subrayados en el plano comunicativo. Podemos sustentar estas afirmaciones sobre la mayor informatividad de la posición posverbal con la observación de algunas cuestiones discursivo-pragmáticas y textuales.

\section{La informatividad textual o discursiva. Algunas precisiones teóricas y su aplicación a la expresión (preverbal o posverbal) y omisión del sujeto en español}

El concepto de informatividad se relaciona con el nivel de procesamiento mental que requiere un texto en función del grado de novedad o de imprevisibilidad que capta en sus receptores (Beaugrande y Dressler, 1997: 201), esto es, alude al nivel de conocimiento que se tiene del mismo, pero, aún más importante, al esfuerzo que implica su comprensión. Normalmente la informatividad se relaciona con el contenido del texto, que a su vez se explica por el nivel de coherencia en la textualidad; se deduce que la coherencia es la que determina si un texto es más o menos informativo en detrimento de otros elementos considerados auxiliares desde un punto de vista textual, como el fonético o el morfológico. Estos elementos no están en el foco de atención de los inter-

11 El estudio está basado en sujetos no pronominales. 
locutores; lo que hace que un texto se sitúe en dicho foco es la coherencia como nivel textual, y no la gramática por sí misma. Por lo tanto, no es que un contenido informativo se presente como información nueva o imprevista, sino que las características semánticas de ese contenido, su selección y su disposición en el texto contribuyen a que haya que emplear un mayor esfuerzo de procesamiento. Con ello, dicho contenido se interpreta como inesperado, imprevisto o nuevo, desplazando la atención sobre esa cláusula o texto, y con ello, el foco informativo.

Hay que señalar que, desde esta perspectiva discursivo-textual, el concepto de foco de atención es claramente diferente al propuesto por la lingüística cognitiva para la prominencia. Beaugrande y Dressler (1997: 201) consideran que la atención es la concentración de la mayor parte de los recursos de procesamiento del sistema cognitivo disponibles para el tratamiento de una única tarea. De este modo, la informatividad de un texto (a la cual se llega por la coherencia textual) está basada también en una focalización de la atención de los hablantes y oyentes. Mientras que la prominencia se basa en el grado de atención que recibe algo activado o accesible para el hablante, la informatividad se corresponde con el grado de novedad o de imprevisibilidad textual; lo menos probable es lo más informativo.

La informatividad, así entendida, presupone un mayor esfuerzo de procesamiento, esto es, a mayor imprevisibilidad, improbabilidad o novedad habrá mayor informatividad, lo que suscita también mayor atención entre los hablantes y oyentes. Beaugrande y Dressler (1997: 42) proponen la comparación entre los ejemplos:

Llámenos antes de cavar una zanja. Probablemente no podrá telefonearnos después.

Llámenos antes de cavar una zanja. Cabe la posibilidad de que haya un cable soterrado. Si usted rompe ese cable, se quedará sin servicio telefónico...

Siguiendo a los autores, el primer ejemplo resulta más informativo que el segundo: el texto presenta el contenido no solamente como nuevo o inesperado, sino que incita al oyente a reflexionar sobre su significado; queda comunicativamente subrayado porque se evocan más posibilidades significativas, ya que no se da ninguna pauta o pista para procesar la razón por la cual el oyente no podrá telefonear, al contrario que en el segundo. Este requiere menos procesamiento que el primero y, aunque incluye más datos sobre la razón de la imposibilidad de acceder a la línea telefónica, este segundo texto, así formulado, capta menos la atención del oyente que el primero; es, por tanto, menos informativo. Procesar un texto o cláusula con más informatividad requiere más atención, y esto puede sugerir paralelamente otros rasgos discursivos y estilísticos como los de solemnidad, argumentación, contundencia, (des)cortesía, realce pragmático, etc. 
Estos significados resultantes son muy útiles para estudiar los fundamentos variables y sociosituacionales del estilo lingüístico ${ }^{12}$.

Según se observa, además, la informatividad es una herramienta de estudio textual que se puede identificar a partir del comportamiento global del texto como vehículo comunicativo; un bajo nivel de informatividad puede provocar la indiferencia del oyente hacia el texto por considerarlo consabido o presupuesto, lo cual, de alguna manera, puede interferir en la efectividad de la comunicación ${ }^{13}$.

En el discurso, la informatividad se presenta de distintas formas y su determinación es compleja. Hay unidades lingüísticas que la potencian o bien la disminuyen; por ejemplo, las palabras funcionales o que marcan relación (artículos, preposiciones y conjunciones) tienen un bajo nivel de informatividad, es decir, aparecen con tanta frecuencia que pasan inadvertidas. Así, la mayor presencia de elementos predecibles y activados o más accesibles atenúa el esfuerzo necesario para procesar la cláusula como conjunto. En los siguientes ejemplos, la introducción de un elemento como desde luego puede reducir la informatividad de Hoy te has portado bien.

Desde luego, hoy te has portado bien

Hoy te has portado bien

En esto no solamente influye la introducción del marcador, sino el significado conjunto del enunciado, así como su inserción en una cláusula y en un texto más amplios. Repercute también la posición relativa de los elementos; nótese que en Hoy te has portado bien, desde luego, la informatividad recae sobre desde luego, lo que paralelamente podría subrayar o reforzar el contenido del segmento precedente.

Estos cambios en el foco suceden porque los textos que se amoldan al conocimiento compartido y conocido por los interlocutores logran menos expectación o atención que aquellos que se presentan como imprevistos o impredecibles (aunque ello no implica que lo sean). Así, una frase como:

12 La interrelación entre texto y situación comunicativa es algo pendiente de explorar, como reconocen los autores (1997: 16). Por otra parte, la informatividad puede verse como un recurso para dar prominencia a elementos que no la tienen: al atraer la atención sobre ellos en un primer momento, se logrará que en cláusulas subsiguientes se vuelvan cognitivamente más prominentes, de modo que la atención pasa del texto a la cognición.

${ }^{13}$ Hay muchas situaciones en la vida cotidiana que, por ser habitualmente conocidas o predecibles por los hablantes, requieren muy poco procesamiento. Una de ellas puede ser, como bien apuntan Beaugrande y Dressler (1997: 15), las instrucciones que se dan en los aviones de cómo ponerse el chaleco salvavidas. Esta sería una situación comunicativa muy poco informativa, pese a la importancia de su contenido. 
Por favor, apaguen las luces al salir

capta menos la atención (o es menos informativa) que si un mensaje similar se configura textualmente de este modo:

Les recordamos la necesidad imperiosa de evitar gastos innecesarios dejando la luz apagada

Esto obedece a que el primer mensaje ya está activado o es accesible para el hablante, ya que se trata de una frase reiteradamente expuesta y de conocimiento compartido. En cambio, la segunda se formula discursivamente de un modo más original a través de la adición de elementos inesperados o novedosos en el mismo contexto. Así pues, una de las manifestaciones más evidentes de la informatividad es la elección de vocablos y construcciones sintácticas que, en conjunto, resulten nuevos, inesperados o imprevisibles para los hablantes y oyentes y que al mismo tiempo evoquen más posibilidades expresivas o de significado, obteniendo así la máxima focalización ${ }^{14}$.

En este sentido, las frases hechas o utilizadas con mucha frecuencia (como por ejemplo Por favor, apaguen las luces al salir), los modismos, los marcadores del discurso, los artículos y las preposiciones propiciarán un menor grado de informatividad que los elementos centrales o nucleares de la predicación, como los sustantivos o los verbos, que se supone concentran la parte más relevante de la comunicación transmitida ${ }^{15}$.

No obstante, medir y predecir la informatividad de un texto no es una tarea fácil; para ella deberían contemplarse numerosos factores más allá de la coherencia. Ha habido algunos intentos de medir la informatividad según probabilidades estadísticas (Shannon y Weaver, 1949), aunque sin demasiado éxito.

\subsection{La informatividad en la variación de los sujetos pronominales}

La idea de que la informatividad discursiva guarda cierta relación con la colocación de las unidades en la cláusula puede aplicarse, obviamente, al estudio de la posición preverbal o posverbal del sujeto en la cláusula (para la variación entre la expresión y la

\footnotetext{
${ }^{14}$ Como bien señalan Beaugrande y Dressler (1997: 76), ser consciente del conocimiento compartido no excluye la creatividad de la comunicación textual, pero sí hace posible que los interlocutores encuentren una orientación para esa creatividad.

${ }^{15}$ Esto conecta, por otra parte, con la idea bastante extendida de que con un número menor de palabras se evocan más ideas que con muchas de ellas. Sin embargo, tal suposición no está exenta de problemas; la selección de las palabras y oraciones de un mensaje y su ordenación deben hacerse cuidadosamente en cada situación comunicativa, para que la comunicación no se vea interferida y para que la interpretación sea la adecuada.
} 
omisión es más relevante la prominencia cognitiva, como se ha visto). El lugar donde se sitúe un elemento sintáctico y la mayor o menor informatividad que pueda adquirir dependerá de variados factores. Sin embargo, de forma general, los estudiosos están de acuerdo en que existe una tendencia a fijar un punto de orientación en el texto antes de presentar información nueva o imprevista; ese punto de orientación es el que permite que la atención se focalice sobre algo nuevo e inesperado (Beaugrande y Dressler, 1997: 128).

Érase una vez un cazador llamado Savari. Estaba muy orgulloso de su fuerza y de su puntería...

En este ejemplo, la atención o focalización la reciben Un cazador llamado Savari y todo lo que sigue, que se ha creado a partir del punto de orientación Érase una vez, el cual constituye una frase prominente $o$ accesible para los oyentes.

Retomando las características de la prominencia señaladas anteriormente, este punto de referencia coincide con el foco de atención o punto de referencia, mientras que el resto de la cláusula lo ocuparía el ámbito de atención o dominio. Como se observa, en la conceptualización de la prominencia el foco es lo que suscita la atención, lo que es más accesible o está activado en la atención o conciencia del hablante. Por el contrario, desde la teoría de la informatividad, lo focal no es ese punto de referencia, sino el resto de la cláusula o el texto, que es destacado o subrayado mediante la atracción de la atención.

Siguiendo a Beaugrande y Dressler (1997: 214), las técnicas de organización de las secuencias lingüísticas constituirían una de las fuentes de expectativas que comparten los interlocutores en la comunicación. De forma habitual, se considera que los contenidos nuevos o inesperados suelen aparecer hacia el final de la cláusula y con una entonación elevada. Por el contrario, los elementos con un bajo nivel de informatividad lo hacen al inicio de las emisiones y presentan una tendencia a ser sustituidos por formas pronominales: Las vacaciones las paso en la playa $>$ Las paso en la playa.

En la misma línea, Firbas (1966) había señalado distintas funciones pragmáticas articuladas en torno a las nociones informativas de tema y rema, que condensan la perspectiva funcional de la frase: el rema es la parte más informativa-comunicativa, mientras que el tema es el punto de partida sobre el cual se construye esa información. Esto puede indicar que, paralelamente, desde un punto de vista cognitivo, el mensaje se estructura como una progresión de la menor a la mayor informatividad (cf. Fried, 2009: 292).

Desde una perspectiva pragmática, Padilla García (2001: 247) considera que las diferencias entre el orden SV y el VS están fundamentadas en la perspectiva que toma el hablante ante las situaciones; la anteposición del sujeto señala la independencia o falta 
de compromiso del hablante con respecto al contenido descrito, mientras que en la posición VS la acción y el sujeto se funden en la perspectiva del hablante.

Anteriormente se señaló que el sujeto posverbal ocupaba la escala más baja de la prominencia. Dado que tradicionalmente se ha establecido que las soluciones pospuestas o finales se reservan para presentar contenidos no esperados, imprevistos, nuevos o no accesibles, todo parecería indicar que, de acuerdo con lo expuesto, un sujeto posverbal tiene un nivel de prominencia menor que sus variantes preverbal y omitida.

En este punto cabría preguntarse si el nivel más bajo de prominencia puede relacionarse con un incremento de la informatividad, entendidos como magnitudes parcialmente opuestas y graduales.

Uno de los indicios que apuntarían a la informatividad de los sujetos pospuestos es, como se sugirió anteriormente, su asimilación a los objetos sintácticos. Algunas investigaciones han concluido que los tipos de verbo con mayor tendencia a la posposición del sujeto son los que pertenecen a las categorías semánticas de reacción psicológica o de función presentativa, tales como aterrar, gustar, entristecer, espantar, emocionar, importar, interesar, ser probable, parecer, resultar, así como algunos intransitivos como aparecer, continuar, durar, existir, ocurrir o llegar (Morales, 1982: 208). A conclusiones parecidas llega Bentivoglio $(1988,1989)$ añadiendo los verbos copulativos a la lista ${ }^{16}$. Sin embargo, un análisis de la naturaleza de los verbos con usted/ustedes pospuesto muestra que este se da con una alta frecuencia cuando este es transitivo o copulativo (87\%) (cf. Serrano, 2012).

Esta elevada frecuencia entre verbos transitivos podría apoyar la idea de que el sujeto pospuesto, al ocupar la posición sintáctica típica de los objetos, asume alguna de sus características y al mismo tiempo se aparta del prototipo de transitividad prototípica. En otro estudio (Serrano, 2012) se apunta a que el esquema VS se aproxima a un modelo de transitividad baja o no prototípica, ya que comparte algunas de las funciones señaladas por Hopper y Thompson (1980: 252), tales como:

a) El sujeto no constituye el origen del evento, sino su término.

(2) Lo están viendo ustedes ahora mismo \se caía el techo de este baño \pero esta no es la única zona afectada \no se muevan porque en unos momentos se lo mostraremoslen Canarias Directo $\backslash(C C E C<M a E n C a n d i r>)^{17}$.

\footnotetext{
${ }^{16}$ La investigación estudia los sujetos no pronominales.

17 Ejemplos extraídos del Corpus Conversacional del Español de Canarias (CCEC).
} 
b) No hay acción o transferencia del agente hacia el paciente. Dado que el agente y el paciente ocupan la posición posverbal, la transferencia del argumento es menos intensa.

(3) Tiene inquietudes en el mundo de la músicalsiempre las ha tenido $\backslash$ en el campo de cantautor presenta usted Itanto aquí como en Madrid una especie de formato nuevo \Canarias pueblo Canarias canción\y también toca otras especialidades \por llamarlo asílfue la época de los $S$. ¿qué recuerdos tiene de esa época?/ (CCEC $<$ MaDiTag $>$ ).

c) De acuerdo con lo anterior, se puede decir que la acción no es puntual o que la transferencia del argumento es transicional, aunque ello dependerá del tipo de verbo y del resto de los elementos de la cláusula.

(4) Ya de hecho están ustedes con el marlel patrimonio de todos los canarios $\backslash(. .) l$. que es esa labor de divulgación lo han estado ustedes repitiendo $\backslash(\mathrm{CCEC}<\mathrm{MaDi}$ RoyCo $>$ ).

d) La agentividad se reduce, no hay afectación del objeto (o no en el mismo grado que en la configuración prototípica SVO); lo afectado es el sujeto que asume la posición de objeto.

(5) Quizás no sea un día para la poesíalpero reconoce usted que el premio Nobel Pablo Nerudaly se atrevería usted a recitarnos algunos de sus poemas?/ (CCEC $<$ MaDiTag $>$ ).

La cercanía sintáctica de los sujetos posverbales a los objetos de las cláusulas con verbo transitivo certifica su condición de contenido objetual. De hecho, la transitividad se concibe como el paso de un flujo o corriente de energía del agente hacia el paciente; es lo que Langacker (1991: 285-286) denominó modelo de evento canónico. En las construcciones prototípicas SVO el agente (fuente) ejerce una acción sobre el paciente (meta) transmitiendo el flujo a través del lexema verbal. Así, en el ejemplo María come peras, que presenta el esquema prototípico SVO, el flujo de energía pasa de María (agente) a peras (paciente) a través del verbo come.

La percepción de esa corriente dependerá de los participantes en la escena y de su ordenación en la cláusula, de modo que en el orden SVO el flujo será mayor que cuando se altera ese orden básico. Así pues, la inversión de los valores prototípicos de agentividad y afectación en VSO puede dar lugar a una disminución del flujo de energía, de modo que el agente pierde buena parte de sus propiedades y se asimila más al paciente. Retomando el ejemplo anterior, si el sujeto se presenta pospuesto: Come peras María, el flujo de energía disminuye, ya no hay acción del agente sobre el paciente, y el sujeto se asimila a esta última noción al ocupar el lugar sintáctico destinado para los objetos. Así pues, la posición preverbal incrementa el flujo de energía y su propia agentividad o 
responsabilidad en el proceso, mientras que la solución pospuesta apuntaría a una reducción de la agentividad, lo cual puede incidir en su afectación y objetualización.

En suma, estos rasgos presentes en el sujeto posverbal se corresponden con unos contenidos nocionalmente concebidos como resaltados, nuevos o inesperados, perfectamente compatibles con la transmisión de informatividad.

Por tanto, el sujeto posverbal posee la menor prominencia con respecto al posverbal (y al omitido), pero, inversamente, es el más informativo de los tres. Por lo tanto, al esquema 1 se puede añadir la colocación del sujeto expreso. Como puede observarse, el sujeto preverbal ocupa el nivel intermedio de esa gradualidad entre la prominencia y la informatividad.

\begin{tabular}{cccc}
\hline & Sujeto omitido & Sujeto preverval & Sujeto posverbal \\
\hline Prominencia cognitiva & + prominente & +/- prominente & - prominente \\
\hline Tabla 2. Gradualidad de la prominencia en las tres variantes del sujeto
\end{tabular}

Tabla 2. Gradualidad de la prominencia en las tres variantes del sujeto

Y se puede realizar otra escala en referencia a la informatividad textual.

\begin{tabular}{lccc}
\hline & Sujeto omitido & Sujeto preverval & Sujeto posverbal \\
\hline Informatividad textual & - informativo & $+/$ - informativo & + informativo \\
\hline
\end{tabular}

Tabla 3. Gradualidad de la informatividad en las tres variantes del sujeto

3. De la cognición al discurso. La conexión entre la prominencia cognitiva y la informatividad textual. El desplazamiento del foco de atención al foco de información

De acuerdo con lo anterior, es posible relacionar la noción cognitiva de prominencia con la de informatividad textual para basar en ellas el estudio de la variación de los pronombres de sujeto. Aunque proceden de distintos enfoques hermenéuticos, comparten algunas características básicas de aplicación discursivo-pragmática. Una de ellas, y quizá la más importante, es la noción de foco. En la conceptualización de la prominencia, el foco de atención recae en la mente del hablante sobre su conocimiento de los referentes en el discurso. Esto hace que, en principio, si un sujeto no se formula, será porque está activado o es accesible cognitivamente; $y$ a la inversa, su eventual formulación recubrirá alguna motivación estilística, sociosituacional, discursivo-pragmática, etc. La prominencia va decayendo según el sujeto va desprendiéndose de la activación mental y se textualiza; por eso un sujeto omitido será más prominente que uno preverbal, y este aún más que el posverbal, que ocupará el nivel más bajo, como ya se ha señalado. El foco de atención prominente en el sujeto posverbal es el más bajo de la escala, pudiendo llegar a ser mínimo o inexistente. 
Por el contrario, cuando el sujeto se expresa, su presencia en el discurso podrá corresponderse también con alguna razón discursiva, como la informatividad, que a su vez dará lugar a distintos significados interactivos. El sujeto posverbal adquiere el mayor foco de información, que va disminuyendo en el preverbal y es menor en el omitido.

Se puede proponer que en esta escala de prominencia/informatividad se produce un desplazamiento del foco de atención (+prominente) al foco de información (+informativo), lo que ocurre de forma gradual entre las tres variantes.

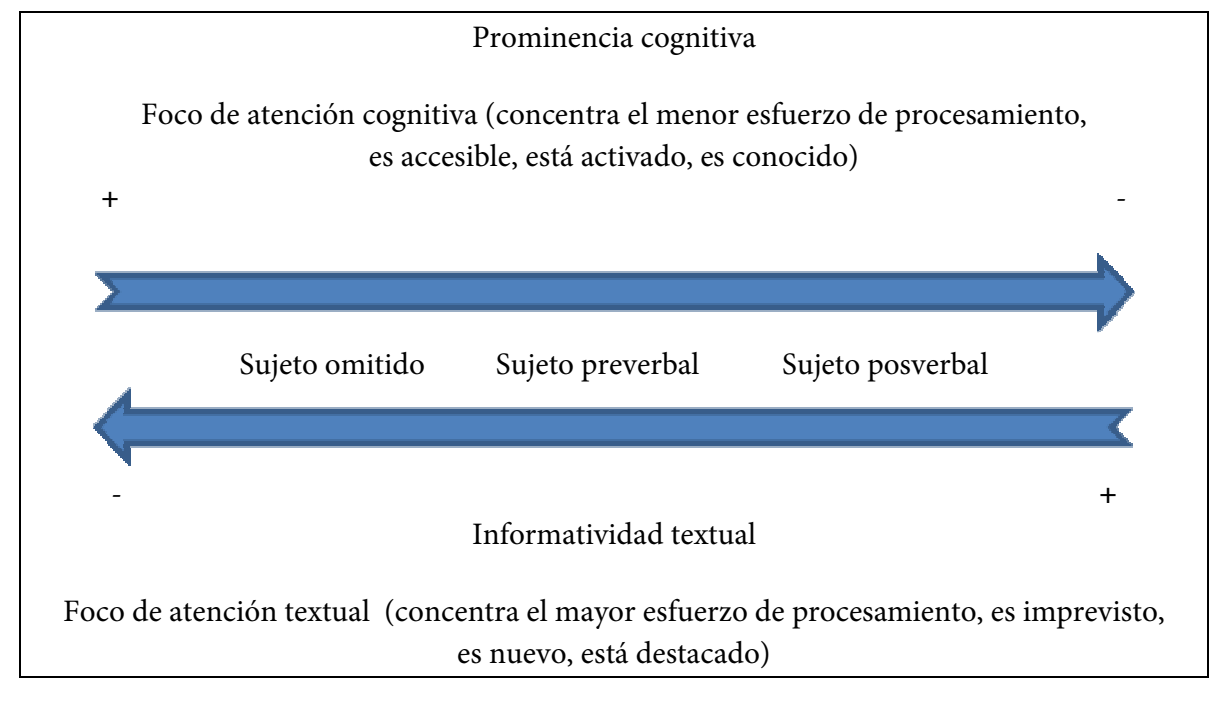

Gráfico 1. Gradualidad de la prominencia cognitiva y la informatividad

Los resultados de algunas investigaciones sobre la expresión/omisión del sujeto y su colocación preverbal/posverbal han podido confirmar la distinta gradación entre la prominencia cognitiva y la informatividad textual, que dan lugar a distintos significados en el discurso y en la interacción comunicativa.

Dichas nociones han sido relevantes para determinar el significado discursivopragmático que adquieren las variantes en la interacción comunicativa a partir de sus fundamentos cognitivos, y que puede dar lugar a un estilo comunicativo creado en conjunción con los elementos sociosituacionales que coaparecen con las variantes. La prominencia cognitiva y la informatividad textual se entienden como un continuum gradual, de forma que las tres variantes del sujeto pronominal participan en mayor o menor medida de ambas nociones, según los esquemas propuestos. 
La variación entre la expresión y la omisión (yo creo/creo, usted(es)/ø, nosotros/ ø) se ha estudiado de acuerdo a la prominencia cognitiva, mientras en que los casos de colocación preverbal o posverbal fueron aplicadas ambas nociones. Esto sucede así porque la posición preverbal, que constituye la categoría intermedia en la escala de prominencia-informatividad, participa simultáneamente de las dos categorías.

La aplicación de las nociones de prominencia cognitiva e informatividad textual ha podido explicar satisfactoriamente las propiedades cognitivas que subyacen a la variación. A continuación se presentan sucintamente algunos resultados obtenidos con respecto a la expresión/omisión de la primera y la segunda persona del paradigma, así como a su colocación preverbal o posverbal.

3.1. La expresión/omisión de la primera y segunda personas pronominales (yo, usted/ustedes, nosotros)

A través de un estudio cualitativo y cuantitativo sobre la expresión del sujeto de primera persona ( $y o$ ) en las construcciones (yo) creo en textos de dos corpus de los medios de comunicación ${ }^{18}$, se comprobó que la expresión del pronombre es más frecuente en aquellas situaciones comunicativas como los debates políticos, donde predomina la función argumentativa.

(6) La gente que venía de la Península no sabía valorar un trajelyo creo que las personas famosas \que vienen aquí al Carnaval deberían de ser invitados\yo creo que podía haber un|||un jurado más específico sobre el tema que estamos tratando $\backslash$ (CCEC $<$ MaTe09>CarnI).

Por su parte, la omisión ( $\varnothing$ creo) es más frecuente en proposiciones donde se produce la formulación de hipótesis y contenidos generales, como se observa en el siguiente ejemplo.

(7) B: Ella tuvo una neumonía en junio|||y estuvo quince días en casal

A: Yo por lo que he leído en prensaltengo la idea de que tu madre\|dejó escrito algo

C: No sabemos $\$ creo que fue algo sobre un dinero que le debia $\backslash$ para que se le pagara $\backslash(C C E C<M a T e 09>)$

Esta variación se produce gracias a las conceptualizaciones cognitivas expuestas: el sujeto omitido es prominente y accesible, lo cual lo hace idóneo para la formulación de

18 Corpus de Lenguaje de los Medios de Comunicación de Salamanca y Corpus Conversacional del Español de Canarias. 
tales contenidos y en las situaciones sociocomunicativas apropiadas, como entre aquellos hablantes que intentan transmitir una imagen de objetividad profesional, como los periodistas. El grupo socioprofesional de los políticos se inclina por la forma con el sujeto expreso yo creo para elaborar sus argumentos. En cuanto a la variación según el sexo/género, encontramos diferencias en cuanto a la frecuencia total de aparición de la forma creo, lo que puede apuntar a distintas orientaciones epistémico-evidenciales en la interacción discursiva (Aijón Oliva y Serrano, 2010b).

Sobre el pronombre usted/ustedes y sus variantes expresa y omitida hemos encontrado algunos resultados similares fundamentados en estas nociones (Serrano y Aijón Oliva, 2010b). Se observa que la expresión del sujeto es más frecuente en aquellos ejemplos donde se desea resaltar su responsabilidad en la acción descrita; en el siguiente ejemplo se coloca el pronombre usted en posición posverbal: Presenta usted, indicando como responsable a la segunda persona de la acción descrita, por lo tanto queda subrayado pragmáticamente en virtud de la informatividad que conlleva.

(8) Presenta usted una nueva denuncia con su sentencia || $\mid$ insistir en que se ejecute la sentencialen el año 2000 fue?/en el año que puso usted la denuncialpues esa sentencia que tiene usted del 2003 intente que se ejecute por todos los medios posibles yendo a ese juzgadolque se investigue si este señor tiene sueldo $\backslash$ salario o pensiones $\backslash($ CCEC $<$ Ma10ElEnv $>$ ).

Por su parte, la expresión en posición preverbal puede dar lugar a significados relacionados con la cortesía, como cuando se quiere dignificar al referente del sujeto haciéndolo presente en la escena comunicativa por medio de la expresión.

(9) Usted conoce profundamente La Laguna \usted ha sido un estudioso de La Laguna $\backslash$ (...) usted lo relataría con cierto toque de ironía \pero sin que se le escape nadalel argumento es muy lagunero ¿no?/ (CCEC $<$ MA1020min $>$ ).

En cambio, la omisión puede servir como un recurso expresivo para difuminar dicha presencia en el contenido descrito, como cuando se tratan temas comprometidos o controvertidos para el interlocutor, como lo es el ejemplo siguiente, donde este interroga a su entrevistada sobre el fallecimiento de su hijo. La omisión del sujeto en ambas cláusulas interrogativas evita focalizar sobre ella para que responda a las preguntas.

(10) ¿Cómo se enteraron $\emptyset$ ?/ (del fallecimiento de su hijo)¿Sabe Ø cuándo va a ser el funeral?/¿Le ha llamado el Presidente Zapatero? \(CCEC $<M a 10 R e>)$.

De hecho, la expresión del sujeto produce un significado menos epistémico que la omisión y conduce a una mayor subjetividad que esta última, porque la predicación se centra en los sujetos. La omisión, en virtud de la prominencia cognitiva, conduce a la creación de estilos más tendentes a la objetividad. Al igual que en la investigación ante- 
rior, encontramos más casos de usted/ustedes omitido entre aquellos hablantes y situaciones que en general requieren la adopción de este estilo comunicativo, como los periodistas y los programas de entretenimiento. Por su parte, los políticos y el género textual de los debates son más proclives a la elaboración de estilos más subjetivos.

El caso del pronombre nosotros revela un comportamiento discursivo diferente al de las otras variantes estudiadas. En textos conversacionales del Corpus Conversacional del Español de Canarias hemos encontrado que se trata de la variante con mayor tendencia a la omisión y que adquiere significados en el discurso que no aparecen (al menos con la misma frecuencia) en la variante expresa ${ }^{19}$. Este pronombre no tiene un referente definido en la lengua española, ni se trata exactamente del plural de la primera persona del paradigma. En esta investigación (Serrano, 2011b) se obtienen dos significados de esta forma: una que incluye al interlocutor y a la audiencia (' $y o+$ otras personas excluyendo la audiencia, tú/vosotros/ustedes) y otra que lo incluye ('yo + otras personas incluyendo la audiencia, tú/vosotros/ustedes). Estas dos posibilidades deícticas se producen con mucha mayor frecuencia en la omisión y más escasamente con la expresión.

La omisión del sujeto constituye una opción comunicativa para el hablante, esto es, si bien en algunos casos la omisión parece obligatoria por la propia prominencia del referente, en otros el hablante tiene la opción de expresarlo u omitirlo, consiguiendo con la expresión un significado mucho más concreto en el discurso que el que aporta la variante omitida.

En el siguiente ejemplo se habla de la admisión de inmigrantes y de la incapacidad para proveerles de empleo. En él, el sujeto nosotros aparece omitido; se supone que se refiere al conjunto de ciudadanos, a los habitantes de Canarias ('yo y todos los demás ciudadanos'), que no podemos darles trabajo.

(11) Pero chica $\emptyset$ no podemos $\backslash$ que no hay trabajo para los de aquí $(C C E C<E l E n 08>)$.

En este otro ejemplo extraído de una asamblea de trabajadores el referente es más concreto, pero, al igual que en el ejemplo anterior, resulta prominente en el discurso, de modo que no hay necesidad de formularlo.

(12) Y lo que sí hemos hecho ø es comentar el paso de T. como delegada sindical por la baja de J.C. $\backslash($ CCEC $<$ ElEn08>).

19 Por otra parte, esto da cuenta de la escasa utilidad que tiene desechar los contextos que no son alternantes en el estudio de la variación morfosintáctica. 
Por el contrario, cuando el pronombre se expresa, cabe suponer que su referente posee un nivel más bajo de prominencia con respecto al omitido, pero su formulación se ve inducida por alguna necesidad o intención discursivo-cognitiva y pragmática que se pone de manifiesto en la interacción y que, paralelamente, conlleva un incremento de su informatividad.

(13) Que nosotros hemos tomado decisiones en reuniones $y$ después el resto de la gente no está informada de lo que hay que hacer $($ CCEC $<$ ElEn08>).

En este caso, la prominencia cognitiva ha permitido confirmar la idea de que la variación sintáctica conlleva siempre el enfoque cognitivo de distintos significados. Ello se proyecta en las conversaciones cotidianas como una posibilidad de expresión de ideas epistémico-evidenciales, seguras o neutrales a partir de los distintos valores de inclusión/exclusión del referente en la omisión.

\subsection{La colocación preverbal/posverbal}

La informatividad textual comienza a percibirse a partir del sujeto expreso y llega a su máximo nivel cuando el sujeto adopta la posición posverbal, como se apuntó anteriormente. De acuerdo a lo expuesto en apartados anteriores, el pronombre usted/ustedes y su posición con respecto al verbo muestra valores opuestos en torno a la gradación entre prominencia e informatividad. La posición preverbal es la prototípica y, en contraste con la posverbal, adquiere un mayor nivel de prominencia cognitiva que esta y uno más bajo de informatividad. Dado que se trata de una variante del sujeto expreso, dicha prominencia conlleva un significado basado en un incremento de la agentividad del sujeto sobre la acción verbal:

(14) La crisis sucesoria que usted intenta negar es absolutamente necesaria en el partido socialista\porque tendrán que rearmarse para una nueva etapa \ustedes tendrán que rearmarse para un éxito electoral $\backslash(M a E n<E l E n v>)$.

La expresión del sujeto (en este caso, de segunda persona, en esta posición y en virtud de la mencionada agentividad) conduce a presentar el contenido matizado en la perspectiva del hablante, que se hace prominente y protagonista en la escena comunicativa y con ello se asocia a su esfera personal, de donde surge la interpretación del enunciado de forma argumentativa. De este modo, el sujeto expreso preverbal da lugar a una emisión basada en la perspectiva del hablante sobre las situaciones, muy común en contextos interactivos en los que es necesario hacer presente a la segunda persona del discurso, y que, al mismo tiempo, sirve para la expresión de opiniones y argumentos. 
La expresión de usted preverbal introduce una opinión personal basada en la agentividad y prominencia del sujeto.

(15) Bueno\usted además trabaja también perfectamente bien la maderalel broncellos brillantes y los topacios $y$ ha hecho también una incursión en la joyería arquitectónica \ $(M a E n<E l E n v>)$.

Por su parte, cuanto este pronombre aparece en posición posverbal, ocupa la posición sintáctica típica de los objetos, de modo que cabría esperar que asimile alguna de las características de los objetos de las construcciones transitivas sin dejar de exhibir, al mismo tiempo, las propiedades de prominencia/informatividad descritas ${ }^{20}$.Esta posición supone una ruptura del esquema prototípico sujeto-verbo y una consecuente asimilación del sujeto al objeto sintáctico, que cognitivamente podría significar la tendencia de la cláusula a asumir significados informativos a través de dicha posición. Un análisis de los ejemplos con sujeto posverbal del corpus da a entender que esta variante se produce con una alta frecuencia cuando el verbo es transitivo o copulativo.

Los verbos transitivos más frecuentes con sujeto posverbal son tener, hacer y ver. Existe una tendencia de esta posición a coaparecer con verbos transitivos o copulativos, como se observa en los siguientes ejemplos:

(16) Y por la mañanalademás de este excepcional programaltienen ustedes la compañíalde una mujerlexcepcional también $\backslash$ no vean la de cosas que tiene que contarnos $\backslash$ escuchen $\backslash$ escuchen $\backslash_{¿}$ lo apuntaron $\backslash n o$ ? $($ MaEn $<$ Vimige $>$ ).

El polo más extremo de la no agentividad del pronombre usted/ustedes posverbal puede relacionarse, por tanto, con la posición prototípica de la objetualidad sintáctica. Sin embargo, estaríamos ante una transitividad no prototípica o baja. Así pues, la afectación que sufre el sujeto en esta posición sintáctica da lugar a un significado informativo, basado en la reducción de la agentividad, que presupone que la cláusula o todo el texto que lo contiene presente la información como nueva; y que adquiere características nocionales basadas en aspectos como la informatividad-focalización-epistemicidad. Todo ello puede dar lugar a una dimensión general basada en un incremento de la literalidad o la fuerza pragmática de lo expresado. Mediante la focalización en el interlocutor, el significado que adquiere el texto está más orientado a la objetividad, favoreciendo que la serie de instrucciones ofrecidas parezcan reales o consabidas para el oyente.

$20 \mathrm{El}$ pronombre usted/ustedes antepuesto en los textos de los medios de comunicación es menos frecuente que el posverbal. Este pronombre se aparta del comportamiento del resto del paradigma verbal, presentando una mayor frecuencia de aquella posición. En los textos conversacionales del mismo corpus, la posposición constituye solamente un $17.2 \%$ del total. 
(17) Presenta usted una nueva denuncia con su sentencia \|||insistir en que se ejecute la sentencialen el año 2000 fue?/en el año que puso usted la denuncia \pues esa sentencia que tiene usted del 2003 intente que se ejecute por todos los medios posibles yendo a ese juzgado\que se investigue si este señor tiene sueldo \salario o pensiones $\backslash$ (CCEC <MaDiRoyCo $>$ ).

Así pues, la posición preverbal constituiría un caso de transitividad alta o prototípica y la posición posverbal de baja o no prototípica, que al mismo tiempo podrían estar asociadas a una gradualidad de la subjetividad. La posición verbo-sujeto aparece con mayor frecuencia con verbos transitivos y el sujeto adopta la posición del objeto, con lo cual el texto y la cláusula adquieren significados informativos-focalizadores y epistémicos. La alta frecuencia de la posición sujeto-verbo en géneros textuales como los debates políticos, que conllevan un elevado nivel de argumentación y de interacción, indica que esta posición construye un significado basado en la perspectiva del hablante sobre las situaciones, esto es, más subjetivo que la posición verbo-sujeto.

En esta investigación pudo confirmarse la escala de prominencia cognitiva/informatividad propuesta, en la que la posición preverbal es más prominente pero menos informativa que la posverbal. La variación sociosituacional muestra que la posición preverbal es en general característica de los textos de debates políticos, la categoría socioprofesional de los políticos y los hombres, con lo cual estas dimensiones, combinadas con los significados de las variantes, orientarían el estilo construido hacia el polo de la subjetividad (Serrano, 2012).

La gradualidad de la prominencia y la informatividad de la colocación preverbal o posverbal puede manifestarse asimismo en significados relacionados con la cortesía verbal. Ello, además de incrementar la fuerza ilocutiva de la emisión, podrá suponer una estrategia para dignificar la imagen de dicho referente cuando el contenido de la cláusula le atribuya una acción o cualidad considerada positiva.

A partir del significado descrito para la posición preverbal, el referente del pronombre de sujeto expreso se hace presente explícitamente en la escena comunicativa, lo cual puede constituir un recurso estilístico de cortesía dignificadora, al asumir la responsabilidad del contenido proposicional.

(18) A: Yo no digo que tengan mala fe $\backslash$ pero la forma $\backslash$ el motivo \lejos de ayudarla, la están hundiendo más yo es lo que digo lyol| esa gente no vale nada|pero una cosa que me enseñaron a mí es el respeto hacia la gentel y a la piba la tiene:::n $\backslash$ amargada (...) en el mundo hay de todo|hay de toda la gama que tú quieras \gordo\flaco \lo que tú quieras (CCEC $<$ MaEn08>).

Por lo que respecta a la posición posverbal, el aumento de la informatividad que lo caracteriza puede conllevar la percepción de una mayor afectación. Con cierta frecuen- 
cia, los casos de sujeto posverbal se producen en contextos peyorativos a partir del carácter objetual que sugiere esta posición, que puede servir para proteger la imagen del interlocutor a partir de la autopeyoración. Así, por ejemplo, el sujeto de primera persona $(y o)$ en posición posverbal puede constituir un recurso de autopeyoración, al centrar la responsabilidad del evento en el referente del sujeto.

(19) A: claro/ claro/ a ver porque/ me imagino yo que como ella ya laseguridad social le empieza a pagarles a ellos yo creo que pronto le empieza a pagar la seguridad social a ellos $\backslash$

B: sí/ yo creo que sí

A: sî la seguridad social paga una parte proporcional

B: ¿aunque estés de baja?/

A: o te manda una carta de despido y tú ya empiezas a negociar/son 25 días por año/(CCEC $<$ MaPly0307>)

De todos modos, estos valores estarán en función de la intención comunicativa del hablante y de la situación de la enunciación, que serán los que finalmente determinarán el estilo comunicativo de (des)cortesía (cf. Serrano y Aijón Oliva, 2010a).

\section{Conclusiones}

Los factores cognitivos y textuales que influyen en la tres variantes del sujeto pronominal en español (expreso preverbal, expreso posverbal y omitido) permiten proporcionar una base interpretativa sólida para definir el significado que se crea con cada una de ellas. Como propiedades opuestas, la prominencia cognitiva y la informatividad textual, aunque proceden de constructos teóricos diferentes, constituyen el eje sobre el cual se pueden establecer los distintos significados y valores de las variantes creados en la interacción comunicativa, que paralelamente podrán dar lugar al desarrollo de estilos comunicativos diferenciados (Serrano y Aijón Oliva, 2011). Hemos querido destacar la importancia del análisis de las propiedades internas de la gramática que subyacen a todo fenómeno de variación como paso indispensable para poder comprender su distribución social y estilística.

Recibido: 26-IV-2012

Aceptado: 14-II-2013

\section{Referencias bibliográficas}

Aijón Oliva, M. A. y Serrano, M. J. (2010a): "Las bases cognitivas del estilo lingüístico", Sociolinguistic Studies 4, 1, págs. 115-144. 
Aijón Oliva, M. A. y Serrano, M. J. (2010b): "El hablante en su discurso: expresión y omisión del sujeto de creo", Oralia 13, págs. 7-38.

Aijón Oliva, M. A. y Serrano, M. J. (2012): “Towards a comprehensive view of variation in language: The absolute variable”, Language \& Communication 32, págs. 80-94.

Alonso Cortés, A. (2002): Lingüística. Madrid, Cátedra.

Ariel, M. (1990): Accessing noun phrase antecedents. Londres, Routledge.

Beaugrande, R. y Dressler, W. U (1997): Introducción a la Lingüística del texto. Barcelona, Ariel.

Bentivoglio, P. (1988): "La posición del sujeto en el español de Caracas: un análisis de los factores lingüísticos y extralingüísticos". En Hammond, R. M. y M.C. Resnick (eds.), Studies in Caribbean Spanish Dialectology. Washington, D.C., Georgetown University Press, págs. 13-23.

Bentivoglio, P. (1989): "Función y significado de la posposición del sujeto nominal en el español hablado", en Estudios sobre el español de América y lingüística afroamericana, Bogotá, Instituto Caro y Cuervo, págs. 40-57.

Chafe, W. L. (1976): "Givenness, contrastiveness, definiteness, subjects, topics, and point of view". En Li, C. (ed.), Subject and Topic. New York, Academic Press, págs. 25-55.

Croft, W. y Cruse, A. (2008): Lingüística Cognitiva. Madrid, Akal.

Crystal, D. (1985): A dictionary of linguistics and phonetics. New York, Blackwell.

Delbecque, N. (2005): "El análisis de corpus al servicio de la gramática funcional y cognoscitiva. Hacia una interpretación de la alternancia sujeto-verbo/verbo-sujeto". En Knauer, G. y V. Bellosta von Colbe (eds.), Variación sintáctica: un reto para las teorías de la sintaxis. Tübingen, Niemeyer, págs. 51-74.

Dik, S. C. (1981): Functional grammar. Dordrecht, Foris.

Evans, V. y Pourcel, S. (2009): New directions in cognitive linguistics. Amsterdam, Benjamins.

Firbas, J. (1966): "Non-thematic subjects in contemporary English", Travaux Linguistique de Prague 2, págs.239-256.

Fried, M. (2009): “Word order”. En Frank, B., J. O. Östman y J. Verschueren (eds.), Grammar, meaning and Pragmatics. Amsterdam, Benjamins, págs. 289-300.

García, E. C. (2009): The motivated syntax of arbitrary signs. Cognitive constraints on Spanish clitic clustering. Amsterdam, Benjamins.

Geeraerts, D., Dirven, R. y Taylor, J. J. (2006): Cognitive Linguistics: Basic readings. Berlín, Mouton de Gruyter.

Gili, Gaya, S. (1976): Curso superior de sintaxis española. Barcelona, Biblograf.

Giora, R. (2003): Salience, context and figurative language. Oxford, University Press. 
Givón, T. (2001): Syntax. An introduction. Amsterdam, Benjamins.

Givón, T. (1995): Functionalism and Grammar. Amsterdam, Benjamins.

Gundel, J. K. y Fretheim, T. (2009): "Information structure". En Frank, B., J.O. Östman y J. Verschueren, (eds.), Grammar, Meaning and Pragmatics. Amsterdam: Benjamins, págs. 146-160.

Halliday, M. A. K. (1967): "Notes on transitivity and theme in English" Part 2., Journal of Linguistics, 3, págs. 199-244.

Hopper, P. y Thompson, S. (1980): "Transitivity in Grammar and Discourse", Language, 56, págs. 252-299.

Lakoff, G. (1987): Women, fire, and dangerous things: What categories reveal about the mind. Chicago, Chicago University Press.

Lambrecht, K. (1998): Information structure and sentence form. Cambridge, University Press.

Langacker, R. W. (2004): "Form, meaning and behavior". En Contini-Morava, E., R.S. Kirsner \& B. Rodríguez Bachiller (eds.), Cognitive and communicative approaches to language. Amsterdam, Benjamins, págs. 21-40.

Langacker, R. W. (1991): Foundations of cognitive grammar. Berlín, Mouton de Gruyter.

Langacker, R. W. (1999): Grammar and conceptualization. Berlín, Mouton de Gruyter.

Langacker, R. W. (2009): Investigations in cognitive grammar. Berlín, Mouton de Gruyter.

Levinson, S. (1983): Pragmatics. Cambridge, University Press.

Lockwood, D., G., Fries, P.H. y Copeland, J.E. (2000): Functional approaches to language, culture and cognition. Amsterdam, Benjamins.

López García, A. (2010): Pluricentrismo, hibridación y porosidad en la lengua española. Madrid/Frankfurt, Iberoamericana/Vervuert.

Morales, A. (1982): "La perspectiva dinámica oracional en el español de Puerto Rico". En Alba, O. (ed.) El español del Caribe. Santiago de los Caballeros, RD, Universidad Católica Madre y Maestra, págs. 203-219.

Padilla García, X. A (2001): El orden de palabras en español coloquial. Tesis doctoral, Universidad de Valencia.

Posio, P. (2012): "The functions of postverbal pronominal pubjects in spoken Peninsular Spanish and European Portuguese", Studies in Hispanic and Lusophone Linguistics 5,1, págs. 149-190.

Prince, E. F. (1981): “Toward a taxonomy of given-new information”. En Cole, P. (ed.), Radical Pragmatics. New York, Academic Press, págs. 223-255.

Serrano, M.J. (2011a): "Morphosyntactic variation in Spain”. En Díaz Campos, M. (ed.), The Handbook of Hispanic Sociolinguistics. Oxford, Wiley-Blackwell, págs. 188-204. 
Serrano, M. J. (2011b): “'Otras personas y yo’. Variación socioestilística del pronombre nosotros en conversaciones espontáneas". En Serrano (ed.), Variación Variable. Almería, Círculo Rojo/Ministerio de Ciencia e Innovación, págs. 93-126.

Serrano, M. J. (2012): "El sujeto pronominal usted/ustedes y su posición: variación y creación de estilos comunicativos", Spanish in Context 9, 1, págs. 109-131.

Serrano, M. J. y Aijón Oliva, M. A. (2010a): "La posición variable del sujeto pronominal en relación con la cortesía interactiva”, Pragmalingüística 18, págs.170-204.

Serrano, M. J. y Aijón Oliva, M. A. (2010b): "Sociocommunicative style and its cognitive properties: subject expression and placement of the Spanish subject (usted/ustedes)". En Cognitive Sociolinguistics: Language Variation in its structural, conceptual and cultural dimensions. Landau, Universität Koblenz-Landau, págs. 5070.

Serrano, M. J. y Aijón Oliva, M. A. (2011): "Syntactic variation and communicative style”, Language Sciences 33, págs. 138-153.

Shannon, C. y Weaver, W. (1949): The Mathematical theory of Communication. Urbana, University of Illinois Press.

Talmy, L. (1988): "Force dynamics in language and cognition", Cognitive Science 12, págs. 49-100.

Vega, M. (2005): “Lenguaje, corporeidad y cerebro”, Signos, 38, págs. 157-176. 